\title{
Can the Onset of Neovascular Age-Related Macular Degeneration Be an Acceptable Endpoint for Prophylactic Clinical Trials?
}

\author{
Luísa S.M. Mendonça ${ }^{a, b}$ Emily S. Levine ${ }^{a, c}$ Nadia K. Waheed ${ }^{a}$ \\ ${ }^{a}$ New England Eye Center, Tufts Medical Center, Boston, MA, USA; ${ }^{b}$ Department of Ophthalmology, Federal \\ University of São Paulo, São Paulo, Brazil; ' Tufts University School of Medicine, Boston, MA, USA
}

\section{Keywords}

Prophylactic clinical trials · Clinical trial endpoints .

Age-related macular degeneration (AMD) - Intermediate

AMD $\cdot$ Neovascular AMD

\begin{abstract}
Many studies over the past 20 years have pursued the goal of preventing or deferring progression from early and intermediate age-related macular degeneration (AMD) to advanced AMD. The onset of neovascular AMD has been used as a primary endpoint in some prophylactic clinical trials because it is easy to assess and relatively well-defined. Nevertheless, the use of this endpoint for assessing progression of AMD lacks validation. The aims of this paper are to review the current practice of clinical trials investigating the prevention of progression of early or intermediate AMD to neovascular AMD, so-called prophylactic trials, as well as identify ongoing efforts to standardize endpoints and select the ideal population for such studies.

(c) 2020 S. Karger AG, Basel
\end{abstract}

\section{Introduction}

Age-related macular degeneration (AMD) is one of the leading causes of blindness worldwide [1-3]. There are several reasons for this. There is no therapy available that prevents the development of geographic atrophy (GA), an end-stage manifestation of AMD. Also, while antiangiogenic drugs have dramatically altered the prognosis of neovascular AMD, studies have shown that, in the long term, patients lose vision because of scarring and macular atrophy [4]. The SEVEN-UP study found a mean loss of 8.6 letters over a mean of 7.3 years (vs. baseline) at entry into the ANCHOR or MARINA trials, and a loss of 19.8 letters over approximately 5 years compared to the visual acuity at the completion of the trials [4].

Clearly, there is still much to be done to achieve better visual results in patients with AMD. Identification of new therapeutic targets may be one path. While testing treatments for the advanced stages of disease, i.e., long-lasting intravitreal drugs, complement inhibitors, and gene therapy, is one approach, it may well be that, once patients are far enough advanced along the AMD pathway, the benefits to be obtained from therapy are limited. Indeed, the karger@karger.com www.karger.com/oph

(c) 2020 S. Karger AG, Basel

Karger"
Nadia Waheed

New England Eye Center 260 Tremont St.

Boston, MA 02116 (USA)

nadiakwaheed@gmail.com 
key for achieving better visual results might lie in the prevention of progression from early and intermediate stages to late AMD. However, this presents a challenging dilemma. Prophylactic trials, which are studies that aim to prevent the development of advanced AMD in eyes at early and intermediate stages, would need to be very long (several years) and very large to achieve adequate statistical power. On the other hand, the endpoints available that can act as surrogate markers for progression and could shorten the duration of such clinical trials, either show a large amount of variability or are imperfect in predicting progression. Overall, both strategies are likely to involve high costs.

This paper focuses on the onset of neovascular AMD as a clinical trial endpoint for prophylactic studies. We discuss how past studies in the field were conducted and identify ongoing efforts to standardize endpoints for such studies. We also look, to a more limited extent, at identifying characteristics that can be used to enrich high-risk populations enrolled in such clinical trials and thereby help reduce the cost of these trials. Finally, we briefly discuss endpoints that can potentially be used as surrogates for neovascular AMD onset, when time to outcome is critical.

\section{Prophylactic Clinical Trial Endpoints: Where Do We Stand?}

For clinical trials of ophthalmic diseases, visual acuity is almost always used as a gold standard endpoint. However, changes in visual acuity are more prominent in the later stages of disease than in the early stages $[1,5,6]$. This makes visual acuity assessment impractical as a measure of progression in intermediate AMD because it may require several years of observation to demonstrate changes $[1,5]$. Thus, an effective surrogate endpoint is needed; this would be one that correlates well with visual acuity but shows changes over a shorter period of time and could therefore be used to reduce the duration of clinical trials. Surrogate endpoints need to be objective, easy to interpret, reproducible, sensitive, and specific, and to correlate well with disease progression and, ultimately, visual acuity. In the context of prophylactic trials of AMD, neovascular onset has been used as a surrogate endpoint by several studies.

The Age-Related Eye Disease Study (AREDS) is a classic and well-known example of a clinical trial designed to assess AMD progression as a primary endpoint. It tested oral antioxidants and zinc as a novel intervention, with the endpoint being progression to advanced AMD, defined either as macular neovascularization (MNV) or center-involving GA [7]. In the pre-optical coherence tomography (OCT) era, macular neovascularization (MNV) onset was diagnosed in this study using color fundus photographs (CFP) $[8,9]$.

Recently, the Intravitreal Aflibercept Injection versus Sham as Prophylaxis against Conversion to Neovascular AMD (PRO-CON) Study (NCT02462889), a clinical trial designed to test the efficacy of aflibercept to prevent highrisk intermediate AMD patients from progressing to exudative $\mathrm{AMD}$, used the proportion of patients converting to neovascular AMD over a 24-month follow-up as its primary outcome using OCT and fluorescein angiography (FA) $[10,11]$. High-risk intermediate AMD was defined in this study as the presence of $>10$ intermediate drusen or 1 large drusen in the study eye and neovascular AMD in the fellow eye [10]. The study failed to prove the efficacy of the therapy (unpublished data); however, as a pioneer study testing invasive therapies for intermediate AMD, it highlighted the importance of proper patient selection including consideration of the risk-benefit ratio for inclusion in interventional prophylactic trials when there is no standard of care for their disease stage.

The Laser Intervention in Early Age-Related Macular Degeneration (LEAD) randomized clinical trial recently tested subthreshold nanosecond laser intervention in eyes with intermediate AMD and bilateral large drusen to prevent progression to advanced stages, either MNV or GA. The trial failed to demonstrate benefits in reducing progression in the overall population. However, it did show an effect modification, in which progression was slowed in eyes without reticular pseudodrusen but increased in eyes presenting with reticular pseudodrusen [12].

The Open-Label Phase 1 Clinical Study to Evaluate the Safety and Tolerability of Subcutaneous Elamipretide in Subjects with Intermediate AMD (ReCLAIM) study (NCT02848313) also enrolled high-risk intermediate AMD patients, defined as having either at least 1 large drusen or multiple medium-sized drusen, in order to test the safety and tolerability of subcutaneous elamipretide which is designed to treat mitochondrial dysfunction secondary to oxidative stress [13]. Because it was a phase I study, efficacy was not assessed, and therefore onset of progression was not an endpoint. A phase II study is now enrolling patients with non-central GA with low-luminance best-corrected visual acuity (LLVA) as its primary endpoint [14]. 
Despite many prophylactic clinical trials defining progression to advanced AMD with a composite primary endpoint (combining the onset of neovascular AMD and GA), such as the AREDS and the LEAD trial noted above, it is important to emphasize that these 2 forms of macular degeneration have separate pathophysiological pathways. Although using a composite endpoint often results in a smaller required sample size and lower study costs, the research question may remain unanswered when a therapy works better for preventing progression to only 1 of the advanced AMD types. Ideally, 3 recommendations need to be met before using a composite endpoint: both components should be similar in importance to the patient, have similar incidences, and be similarly affected by the intervention [15]. Although GA and MNV are equally important complications for the patient, the frequencies and risk factors for the occurrence of these 2 forms of disease are different, as are the treatment options. Henceforth, we discuss here the use of neovascular onset as a primary endpoint but will also cite features associated with GA progression as some are common to both advanced forms of AMD.

There are still no validated surrogate outcome measures or endpoints accepted by regulatory agencies specifically for drug development for intermediate AMD [5]. The currently accepted surrogate endpoints refer to eyes with late stages of disease: GA growth rate for late dry AMD, and fluid on OCT and leakage on FA for neovascular AMD. However, there are ongoing studies aiming to validate endpoints for prophylactic trials in intermediate AMD populations. The MACUSTAR consortium is a multicenter initiative to establish functional, morphological, and self-reported outcomes in intermediate AMD [5]. Morphological assessments used to identify the onset of MNV and GA included spectral domain OCT, CFP, and FA. The IMPACT study (NCT03688243) is a multicenter clinical trial currently enrolling intermediate AMD patients to investigate and establish robust and repeatable anatomic and vascular metrics. The study uses OCT and OCT angiography (OCTA) markers such as choriocapillaris (CC) nonperfusion, drusen volume, detection of nonexudative MNV at baseline, and other structural OCT markers [16].

Progression to neovascular AMD is a promising endpoint for medications that potentially inhibit angiogenic signals. There are several advantages to this endpoint. One, it is relatively unambiguous and easy to assess using traditional imaging modalities that are present in most clinical settings. Second, it has been associated with progression of disease, and ultimately with loss of visual acu- ity $[9,17]$. Thus, it meets most of the criteria required for a surrogate endpoint. It is worth noting that the development of nonexudative MNV can be protective in eyes with atrophy, preventing its enlargement within the area of MNV [18, 19]. Therefore, when choosing surrogate endpoints for loss of function, MNV onset followed by exudation would be the outcome to be used, rather than MNV development in general.

In the context of exudative MNV as a primary endpoint, important design aspects should be considered. Studies need to be carefully powered so as to have a large enough sample size to demonstrate differences in conversion rates with treatment. The AREDS reported a $10 \%$ conversion rate in patients that presented with nonadvanced AMD in both eyes. When considering the fellow eyes of patients with unilateral neovascular AMD, the rate of conversion was around 35\% in a relatively long followup of 6.3 years [20]. In a post hoc analysis of the MARINA and ANCHOR studies, the rates of conversion of fellow eyes were $15.9-26.4 \%$ at 12 months and $23.8-38.8 \%$ at 24 months [21]. An analysis of the fellow eyes of patients enrolled in the Comparison of Age-Related Macular Degeneration Treatments Trials (CATT) revealed an incidence of neovascular AMD of $20.3 \%$ at 24 months [22]. A post hoc analysis of fellow eyes in the HARBOR trial found a $12.2 \%$ progression rate to exudative $\mathrm{MNV}$ at 2 years, based exclusively on SD-OCT analysis of eligible patients [23]. The PRO-CON study, which had as an inclusion criterion the presence of neovascular AMD in the fellow eye, identified progression to neovascular AMD in 9.38 and $10.90 \%$ of eyes in the sham group at months 12 and 24 , respectively [10]. These differences in rates of progression among intermediate AMD patients, as well as the varying times to conversion, highlight the importance of proper choice of inclusion criteria and targeting subjects that are more likely to progress over a given interval.

In most prophylactic clinical trials (e.g., PRO-CON and LEAD), inclusion criteria consider the risk of an eye converting to exudative MNV. This is important to adequately power the study, but is also relevant when invasive therapies are tested. Since many patients will not actually benefit from treatment, considering the risk-benefit ratio for inclusion in a prophylactic trial is important. Several strategies can be employed to enrich the study with subjects who are more likely to convert, which has the dual benefit of allowing for a lower sample size or shorter follow-up duration as well as increasing the benefit-risk ratio to the patients. These strategies are discussed below. 

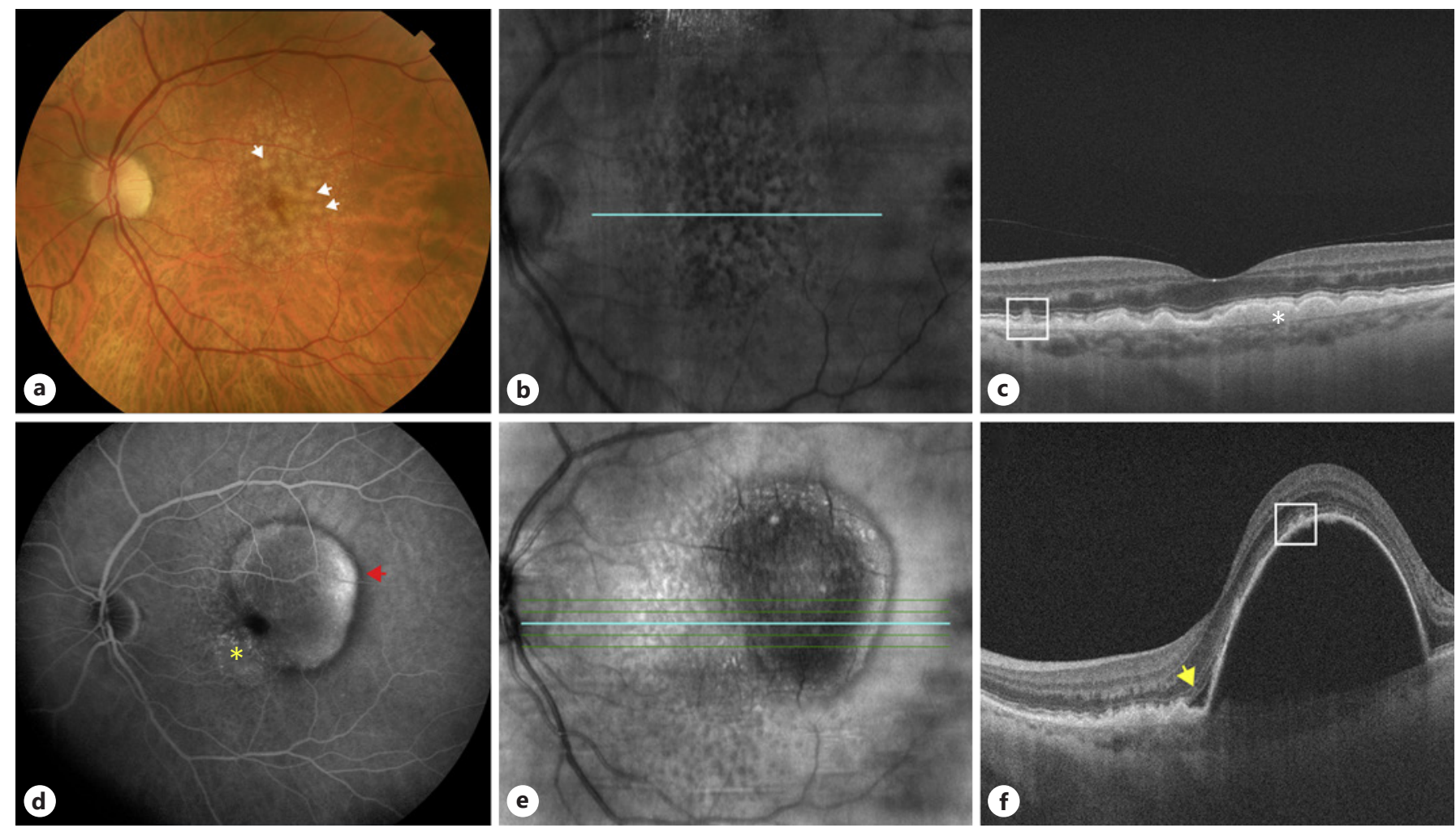

Fig. 1. Intermediate age-related macular degeneration (AMD) (ac) with progression to exudative macular neovascularization $(\mathrm{MNV})(\mathbf{d}-\mathbf{f})$. a Color fundus photo shows numerous large drusen (white arrows) in the macula at baseline. Optical coherence tomography (OCT) en face (b) and B-scan (c) show a subretinal drusenoid deposit (white square), sub-retinal pigment epithelium (RPE) drusen, and drusenoid pigment epithelium detachment (white asterisk) at baseline. d Fluorescein angiography with dye leakage, stippled fluorescence (yellow asterisk) and late staining (red arrow) consistent with a type $1 \mathrm{MNV}$ that developed 24 months after baseline images. OCT en face (e) and B-scan (f) show subretinal drusenoid deposits (white square) above a RPE detachment due to a type $1 \mathrm{MNV}$ with subretinal fluid (yellow arrow).

\section{Optimizing Prophylactic Clinical Trials: Inclusion Criteria Based on Risk Factors}

Several scales that include demographic, environmental, and genetic factors as well as imaging biomarkers to predict progression in intermediate AMD have been published to allow for the selection of high-risk patients for enrollment in such clinical trials [2, 23-27]. Models that assess time-to-progression are particularly useful as references for designing clinical trials that, in general, last 2 years.

Age, family history, smoking status, advanced AMD in the fellow eye, genetic variants (CFH Y402H and ARMS2 A69S), and imaging features like large drusen (Fig. 1) and pigmentary changes on CFP, were found to correlate with progression to advanced AMD, both GA or MNV, in a multivariate analysis [2]. Considering the status of the fellow eye at baseline, patients with MNV in the fellow eye were more likely to progress to $\mathrm{MNV}$, while patients with GA in the fellow eye were more likely to progress to GA [2]. Additionally, conversion to MNV in the fellow eye in the past 2 years was associated with a higher risk of conversion of $\mathrm{MNV}$ in the study eye compared to a conversion that happened $>2$ years prior (unpublished data).

An association between OCT features in intermediate AMD and progression to MNV has also been extensively investigated. Hyperreflective foci (HRF) (Fig. 2), hyporeflective foci within drusenoidlesions, subretinal drusenoid deposits (also called reticular pseudodrusen), (Fig. 1) and RPE thickening have been associated with progression to MNV [3, 23, 28-30].

A recently published state-of-the-art machine-learning study developed predictive models for MNV and GA development in a cohort of intermediate AMD eyes based on OCT features that were identified by means of artificial intelligence (AI) tools combined with demographic 
Fig. 2. Intermediate age-related macular degeneration (AMD) (a-d) with progression to exudative macular neovascularization $(\mathrm{MNV})(\mathbf{e}, \mathbf{f})$. En face optical coherence tomography (OCT) (a) and B-scan (b) with sub-retinal pigment epithelium (RPE) drusen at baseline. En face (c) and B-scan (d) OCT with intraretinal hyperreflective foci (white arrow), corresponding to pigment clump migration into the retina 12 months after baseline images. En face (e) and B-scan (f) OCT after progression to exudative AMD, with subretinal fluid (yellow arrow) and an area of RPE atrophy (black asterisk), 32 months after baseline images.
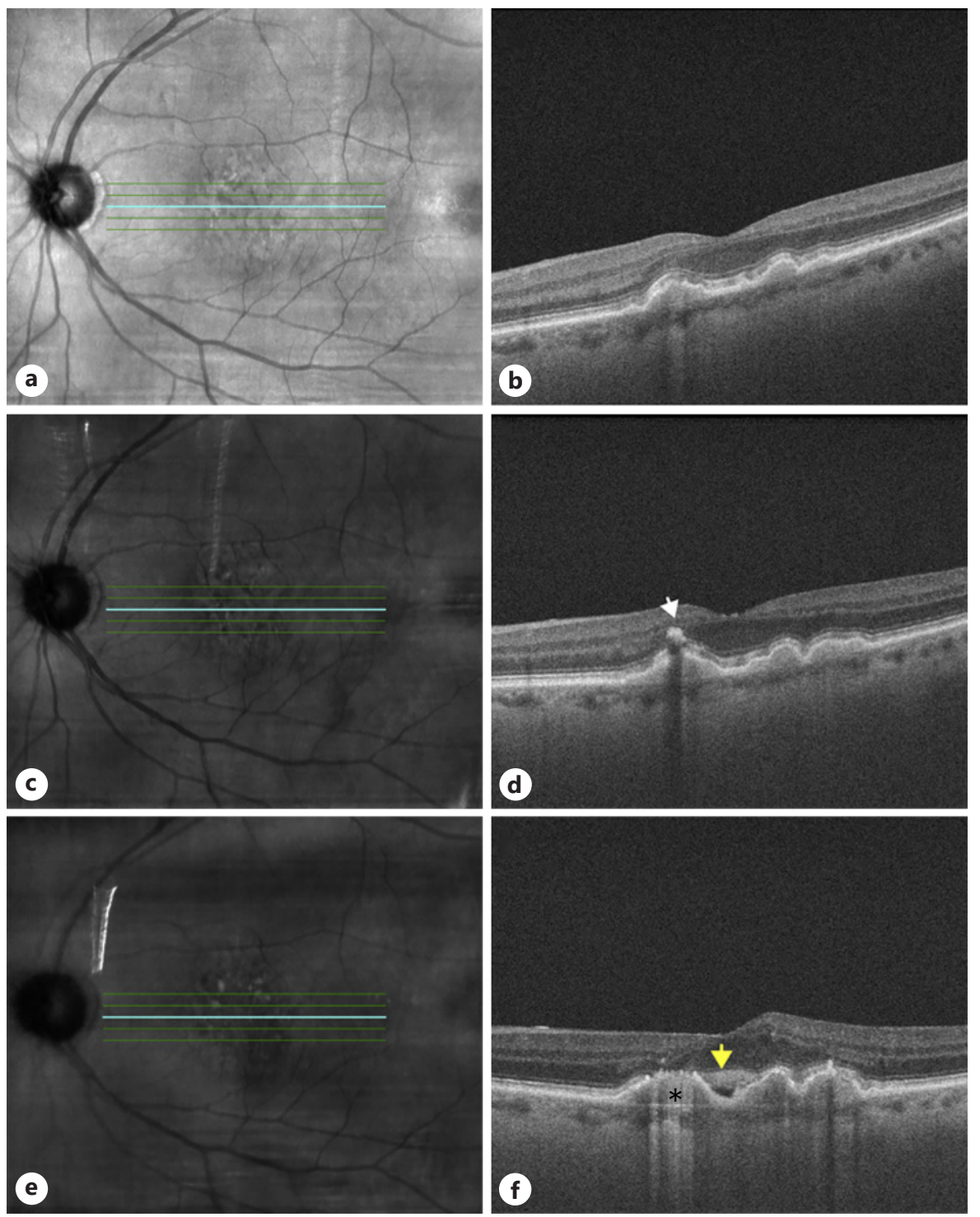

and genetic data. The authors found that drusen-centric metrics (e.g., RPE + drusen volume and thickness, but not drusen volume or area alone) seemed more relevant to the progression to $\mathrm{MNV}$ whereas neurosensory retinal changes and age were more relevant to the development of GA [3]. For both outcomes, hyperreflective foci were highly weighted in the model and represented the second most important feature; this confirmed findings of earlier studies that showed that pigmentary changes correlate with progression to both advanced forms of AMD [2, 3, 31]. Therefore, the presence of hyperreflective foci should be considered a relevant risk factor for AMD progression in general.

Neovascular AMD as an Endpoint for Prophylactic Clinical Trials
Other AI models have been proposed, using either OCT or CFP to predict progression to exudative MNV, with satisfactory accuracy $[32,33]$. Markers used for prediction in these studies are mainly drusen and pigmentary changes on CFP [32], and segmentation of external layers on OCT. Pixels under the RPE were used to identify progressors, as opposed to pixels around the RPE that had a higher impact on identifying nonprogressors as per occlusion sensitivity analysis [33]. More recently, Yim et al. [34] developed a model to predict progression to neovascular AMD within 6 months, using deep learning for performing both a comprehensive feature extraction on OCT and prediction. Interestingly, the authors proposed 
Fig. 3. Nonexudative MNV imaged with optical coherence tomography (OCT) angiography. a En face OCT angiography choriocapillaris slab $(3 \times 3 \mathrm{~mm})$ showing a neovascular complex. b Structural B-scan showing a double-layer sign with flow overlay underneath the retinal pigment epithelium and above Bruch's membrane (white arrows), corresponding to the nonexudative MNV. c Structural en face OCT choriocapillaris slab $(6 \times 6 \mathrm{~mm})$. d Structural B-scan showing a double-layer sign.
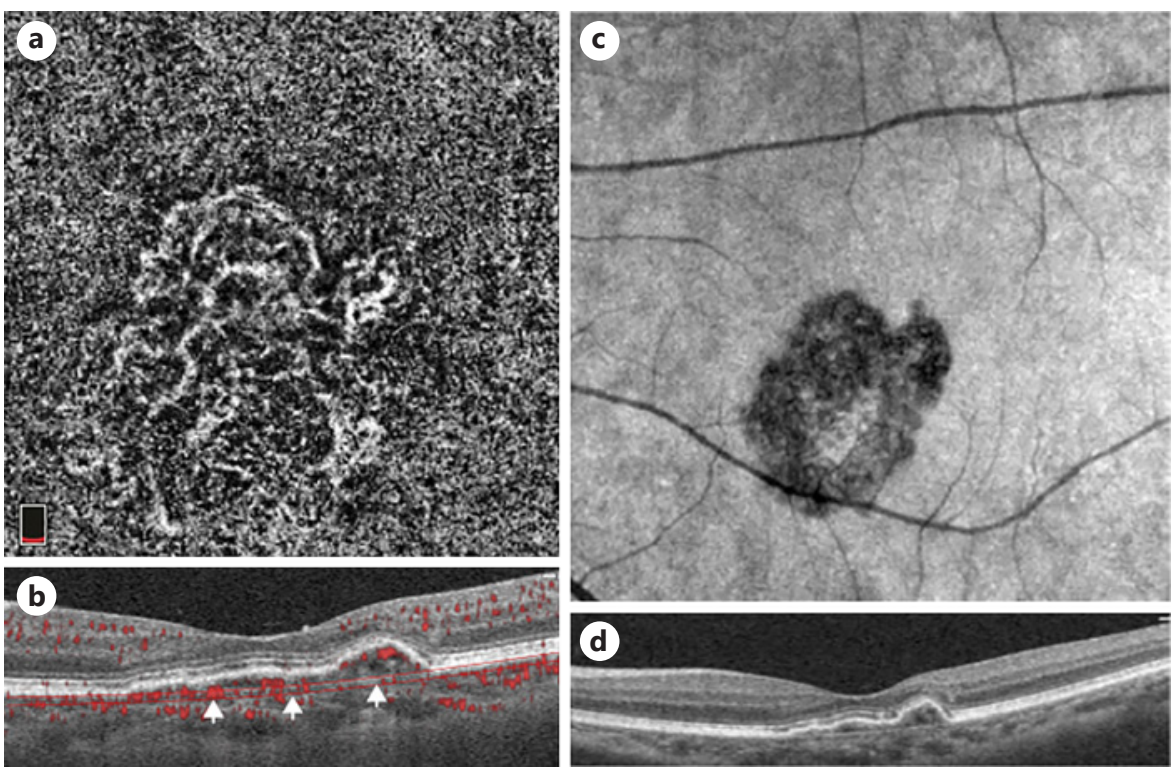

configurable operating points, allowing different balances between sensitivity and specificity.

It has been shown that increases in drusen volume and area in 2 years predicts progression to neovascular AMD $[29,30]$, so the variability of those measures should also be taken in account when selecting patients at risk for progression $[3,29]$. There have been conflicting results in the literature regarding the interval at which drusen volume is capable of predicting progression. The study of de Sisternes et al. [24] showed that drusen volume and area were predictors of MNV development after 24 months; other groups demonstrated the significance of these metrics for predicting progression within 2 years [3, 23, 28, 29]. This discrepancy might be due to differences regarding other risk factors in these populations.

The value of functional metrics, such as microperimetry and low-luminance visual acuity, for predicting progression to advanced AMD has recently been studied. These metrics failed to improve the predictive performance of a model that included pigmentary abnormalities and drusen, as graded on CFP [35].

It is known that eyes with nonexudative or subclinical MNV (Fig. 3) present a higher risk of progression to exudation than do eyes with drusen-only intermediate AMD $[36,37]$. A longitudinal study employing swept-source (SS)-OCTA for detecting nonexudative MNV demonstrated a 15.2-fold increase in the risk of exudation in eyes with this finding versus eyes without nonexudative MNV [36]. Therefore, it is important at the baseline to identify nonexudative $\mathrm{MNV}$ in patients with intermediate $\mathrm{AMD}$ enrolled in prophylactic clinical trials, defining a separate subgroup analysis for this condition, or balancing it between the control and treatment group to ensure a proper interpretation of results. The IMPACT study has, amongst its aims, the purpose of investigating the presence of nonexudative AMD at baseline in eyes otherwise classified as intermediate AMD using SS-OCTA [16]. A standardization of this assessment may be useful for future clinical trials that may adopt OCTA testing at baseline for all eyes with intermediate AMD.

Studies that specifically evaluate the prevention of nonexudative MNV conversion are also needed. The PRO-CON study did not demonstrate reduced progression rates in eyes that had nonexudative MNV, but this trial was not designed or powered for this purpose [10]. It is important to establish in advance how to interpret the development of nonexudative MNV throughout followup, e.g., if this finding is identified as a positive conversion or if the presence of exudation is necessary to mark a conversion, the latter being the better option when using a surrogate endpoint for function as the intended strategy.

\section{Other Potential Surrogate Endpoints in Prophylactic Clinical Trials}

When the duration of the study is a concern, and a shorter follow-up is desired by the sponsor, an alternative approach to using the onset of neovascular AMD may be 
to evaluate progression of drusen as a surrogate endpoint $[1,3,15]$. This would likely be a more sensitive parameter, allowing for the assessment of subtle changes in drusen area and volume prior to the development of clinically identifiable progression, and therefore providing parameters for study of earlier interventions $[1,15]$. This topic is not within the scope of this study and will thus not be addressed in further detail.

Additionally, OCTA emerging as a promising noninvasive tool for MNV diagnosis and follow-up created the need for studying the utility of this modality for future clinical trials. To date, the relationship between increased $\mathrm{CC}$ nonperfusion in intermediate $\mathrm{AMD}$ and progression to exudative MNV has yet to be determined. However, if proven, this could be a target for drug development in intermediate AMD and a surrogate outcome measure that could be observed in earlier stages of disease, replacing the traditional approach of following patients until they develop clinically identifiable MNV. Nevertheless, there are numerous pitfalls to CC analysis, including variability, a lack of standardization across research groups, and the fact that it is very sensitive to erroneous segmentation and inappropriate binarization techniques [3840].

\section{Conclusion}

Prophylactic clinical trials are imperative for changing long-term achievable visual results in AMD. Despite recent efforts, therapies to prevent or defer progression from early and intermediate AMD to advanced stages have not succeeded. Currently, many prophylactic studies use the onset of neovascular AMD as the primary end- point, or a composite primary endpoint that combines progression to GA and neovascular AMD. Based on current knowledge, it is reasonable to state that neovascular onset is an acceptable endpoint for prophylactic trials, as it is easy to assess and relatively well-defined. It can also be enriched using strategies such as identifying eyes with a higher risk for progression, as there is robust literature available on risk factors for progression to neovascular AMD.

\section{Conflict of Interest Statement}

L.S.M.M. and E.S.L. have no conflicts of interest to declare. N.K.W.: Topcon Medical Systems, Inc. (Oakland, NJ, USA) (C, S, R), Nidek Medical Products, Inc. (R), Carl Zeiss Meditec, Inc. (S), Heidelberg Engineering (S), Roche/Genentech (C, S), Regeneron Pharmaceuticals, Inc (C), Apellis (C, S), Astellas (C, S), Boehringer Ingelheim (C, S), Novartis (C, S), Novartis (C, S), Gyroscope (I, S), Boston Image Reading Center (I), Ocudyne (I).

\section{Funding Sources}

The authors report grants from Massachusetts Lions Club and Research to Prevent Blindness. L.S.M.M. reports a research scholarship granted by CAPES, Ministry of Education, Brazil (in the scope of Capes-PrInt program, process No. 88887.369769/2019$00)$.

\section{Author Contributions}

L.S.M.M. performed the literature review and worked on manuscript conceptualization and drafting. E.S.L. worked on the drafting and critical review of the manuscript. N.K.W. performed the literature review and worked on conceptualization, drafting, and critical review of the manuscript.

\section{References}

1 Schaal KB, Rosenfeld PJ, Gregori G, Yehoshua Z, Feuer WJ. Anatomic Clinical Trial Endpoints for Nonexudative Age-Related Macular Degeneration. Ophthalmology. 2016 May; 123(5):1060-79.

2 Klein ML, Francis PJ, Ferris FL 3rd, Hamon SC, Clemons TE. Risk assessment model for development of advanced age-related macular degeneration. Arch Ophthalmol. 2011 Dec;129(12):1543-50.

3 Schmidt-Erfurth U, Waldstein SM, Klimscha S, Sadeghipour A, Hu X, Gerendas BS, et al. Prediction of Individual Disease Conversion in Early AMD Using Artificial Intelligence. Invest Ophthalmol Vis Sci. 2018 Jul;59(8): 3199-208.
4 Rofagha S, Bhisitkul RB, Boyer DS, Sadda SR, Zhang K; SEVEN-UP Study Group. Sevenyear outcomes in ranibizumab-treated patients in ANCHOR, MARINA, and HORIZON: a multicenter cohort study (SEVENUP). Ophthalmology. 2013 Nov; 120(11): 2292-9.

5 Finger RP, Schmitz-Valckenberg S, Schmid M, Rubin GS, Dunbar H, Tufail A, et al.; on behalf of the MACUSTAR consortium. MACUSTAR: Development and Clinical Validation of Functional, Structural, and PatientReported Endpoints in Intermediate Age-Related Macular Degeneration. Ophthalmologica. 2019;241(2):61-72.
6 Wu Z, Ayton LN, Guymer RH, Luu CD. Lowluminance visual acuity and microperimetry in age-related macular degeneration. Ophthalmology. 2014 Aug;121(8):1612-9.

7 Age-Related Eye Disease Study Research Group. The Age-Related Eye Disease Study (AREDS): design implications. AREDS report no. 1. Control Clin Trials. 1999 Dec;20(6): 573-600.

8 Age-Related Eye Disease Study Research Group. A randomized, placebo-controlled, clinical trial of high-dose supplementation with vitamins $\mathrm{C}$ and $\mathrm{E}$ and beta carotene for age-related cataract and vision loss: AREDS report no. 9. Arch Ophthalmol. 2001 Oct; 119(10):1439-52.
Neovascular AMD as an Endpoint for Prophylactic Clinical Trials
Ophthalmologica 2021;244:379-386 DOI: $10.1159 / 000513083$ 
9 Age-Related Eye Disease Study Research Group. A randomized, placebo-controlled, clinical trial of high-dose supplementation with vitamins $\mathrm{C}$ and $\mathrm{E}$, beta carotene, and zinc for age-related macular degeneration and vision loss: AREDS report no. 8. Arch Ophthalmol. 2001 Oct;119(10):1417-36.

10 Heier JS. Prophylaxis Intravitreal Aflibercept against Conversion to Neovascular Age-Related Macular Degeneration in High Risk Eyes (PRO-CON). 24-Month Results. American Society of Retina Specialists Annual Meeting; Chicago, IL; July 27-30, 2019.

11 Heier JS. [Internet] IAI versus Sham as Prophylaxis against Conversion to Neovascular AMD (PRO-CON) 2015 [cited 31 March 2016]. Available from: https://clinicaltrials. gov/ct2/show/NCT02462889.

12 Guymer RH, Wu Z, Hodgson LA, Caruso E, Brassington $\mathrm{KH}$, Tindill $\mathrm{N}$, et al.; Laser Intervention in Early Stages of Age-Related Macular Degeneration Study Group. Subthreshold Nanosecond Laser Intervention in Age-Related Macular Degeneration: The LEAD Randomized Controlled Clinical Trial. Ophthalmology. 2019 Jun;126(6):829-38.

13 Cousins SW. [Internet] An Open-Label, Phase 1 Clinical Study to Evaluate the Safety andTolerability ofSubcutaneousElamipretide in Subjects with Intermediate Age-Related Macular Degeneration 2016 [cited 20 October 2020]. Available from: https://clinicaltrials. gov/ct2/show/NCT02848313.

14 [Internet] ReCLAIM-2 study to evaluate safety, efficacy and pharmacokinetics of elamipretide in subjects with AMD with noncentral GA (ReCLAIM-2) 2019 [cited 28 September 2020]. Available from: https://clinicaltrials.gov/ct2/show/NCT03891875.

15 Garcia Filho CA, Yehoshua Z, Gregori G, Nunes RP, Penha FM, Moshfeghi AA, et al. Change in drusen volume as a novel clinical trial endpoint for the study of complement inhibition in age-related macular degeneration. Ophthalmic Surg Lasers Imaging Retina. 2014 Jan-Feb;45(1):18-31.

16 Waheed N, Rosenfeld P. Non-exudative AMD Imaged With SS-OCT https://clinicaltrials.gov/ct2/show/NCT03688243? cond=int ermediate+age+related + macular + degenerati on \&draw $=4 \&$ rank $=302018$

$17 \mathrm{Hu}$ X, Waldstein SM, Klimscha S, Sadeghipour A, Bogunovic H, Gerendas BS, et al. Morphological and functional characteristics at the onset of exudative conversion in agerelated macular degeneration. Retina. 2020; 40:1070-8.

18 Capuano V, Miere A, Querques L, Sacconi R, Carnevali A, Amoroso F, et al. Treatment-Naïve Quiescent Choroidal Neovascularization in Geographic Atrophy Secondary to Nonexudative Age-Related Macular Degeneration. Am J Ophthalmol. 2017 Oct;182:45-55.

19 Pfau M, Möller PT, Künzel SH, von der Emde L, Lindner M, Thiele S, et al. Type 1 Choroidal Neovascularization Is Associated with Re- duced Localized Progression of Atrophy in Age-Related Macular Degeneration. Ophthalmol Retina. 2020 Mar;4(3):238-48.

20 Clemons TE, Milton RC, Klein R, Seddon JM, Ferris FL 3rd; Age-Related Eye Disease Study Research Group. Risk factors for the incidence of Advanced Age-Related Macular Degeneration in the Age-Related Eye Disease Study (AREDS) AREDS report no. 19. Ophthalmology. 2005 Apr;112(4):533-9.

21 Barbazetto IA, Saroj N, Shapiro H, Wong P, Ho AC, Freund KB. Incidence of new choroidal neovascularization in fellow eyes of patients treated in the MARINA and ANCHOR trials. Am J Ophthalmol. 2010 Jun;149(6): 939-946.e1.

22 Zhou Q, Daniel E, Maguire MG, Grunwald JE, Martin ER, Martin DF, et al.; Comparison of Age-Related Macular Degeneration Treatments Trials Research Group. Pseudodrusen and Incidence of Late Age-Related Macular Degeneration in Fellow Eyes in the Comparison of Age-Related Macular Degeneration Treatments Trials. Ophthalmology. 2016 Jul; 123(7):1530-40.

23 Nassisi M, Lei J, Abdelfattah NS, Karamat A, Balasubramanian S, Fan W, et al. OCT Risk Factors for Development of Late Age-Related Macular Degeneration in the Fellow Eyes of Patients Enrolled in the HARBOR Study. Ophthalmology. 2019 Dec;126(12):1667-74.

24 de Sisternes L, Simon N, Tibshirani R, Leng T, Rubin DL. Quantitative SD-OCT imaging biomarkers as indicators of age-related macular degeneration progression. Invest Ophthalmol Vis Sci. 2014 Oct;55(11):7093-103.

25 Ferrara D, Silver RE, Louzada RN, Novais EA, Collins GK, Seddon JM. Optical Coherence Tomography Features Preceding the Onset of Advanced Age-Related Macular Degeneration. Invest Ophthalmol Vis Sci. 2017 Jul; 58(9):3519-29.

26 Seddon JM, Reynolds R, Maller J, Fagerness JA, Daly MJ, Rosner B. Prediction model for prevalence and incidence of advanced age-related macular degeneration based on genetic, demographic, and environmental variables. Invest Ophthalmol Vis Sci. 2009 May;50(5): 2044-53.

27 Zanke B, Hawken S, Carter R, Chow D. A genetic approach to stratification of risk for agerelated macular degeneration. Can J Ophthalmol. 2010 Feb;45(1):22-7.

28 Folgar FA, Yuan EL, Sevilla MB, Chiu SJ, Farsiu S, Chew EY, et al.; Age Related Eye Disease Study 2 Ancillary Spectral-Domain Optical Coherence Tomography Study Group. Drusen Volume and Retinal Pigment Epithelium Abnormal Thinning Volume Predict 2-Year Progression of Age-Related Macular Degeneration. Ophthalmology. 2016 Jan;123(1):39-50.e1.

29 Lamin A, Dubis AM, Sivaprasad S. Changes in macular drusen parameters preceding the development of neovascular age-related macular degeneration. Eye (Lond). 2019 Jun; 33(6):910-6.
30 Roberts PK, Baumann B, Schlanitz FG, Sacu S, Bolz M, Pircher M, et al. Retinal pigment epithelial features indicative of neovascular progression in age-related macular degeneration. Br J Ophthalmol. 2017 Oct;101(10): 1361-6.

31 Sleiman K, Veerappan M, Winter KP, McCall MN, Yiu G, Farsiu S, et al.; Age-Related Eye Disease Study 2 Ancillary Spectral Domain Optical Coherence Tomography Study Group. Optical Coherence Tomography Predictors of Risk for Progression to Non-Neovascular Atrophic Age-Related Macular Degeneration. Ophthalmology. 2017 Dec; 124(12):1764-77.

32 Burlina PM, Joshi N, Pacheco KD, Freund DE, Kong J, Bressler NM. Use of Deep Learning for Detailed Severity Characterization and Estimation of 5-Year Risk Among Patients with Age-Related Macular Degeneration. JAMA Ophthalmol. 2018 Dec;136(12):1359_ 66.

33 Russakoff DB, Lamin A, Oakley JD, Dubis AM, Sivaprasad S. Deep Learning for Prediction of AMD Progression: A Pilot Study. Invest Ophthalmol Vis Sci. 2019 Feb;60(2):71222.

34 Yim J, Chopra R, Spitz T, Winkens J, Obika A, Kelly C, et al. Predicting conversion to wet age-related macular degeneration using deep learning. Nat Med. 2020 Jun;26(6):892-9.

35 Wu Z, Luu CD, Hodgson LA, Caruso E, Chen FK, Chakravarthy U, et al. Examining the added value of microperimetry and low luminance deficit for predicting progression in age-related macular degeneration. $\mathrm{Br} \mathrm{J} \mathrm{Oph-}$ thalmol.

2020 Jun;bjophthalmol-2020-315935.

36 de Oliveira Dias JR, Zhang Q, Garcia JM, Zheng F, Motulsky EH, Roisman L, et al. Natural History of Subclinical Neovascularization in Nonexudative Age-Related Macular Degeneration Using Swept-Source OCT Angiography. Ophthalmology. 2018 Feb;125(2): 255-66.

37 Treister AD, Nesper PL, Fayed AE, Gill MK, Mirza RG, Fawzi AA. Prevalence of Subclinical CNV and Choriocapillaris Nonperfusion in Fellow Eyes of Unilateral Exudative AMD on OCT Angiography. Transl Vis Sci Technol. 2018 Oct; 7(5):19.

38 Chu Z, Gregori G, Rosenfeld PJ, Wang RK. Quantification of Choriocapillaris with Optical Coherence Tomography Angiography: A Comparison Study. Am J Ophthalmol. 2019 Dec;208:111-23.

39 Chu Z, Zhang Q, Zhou H, Shi Y, Zheng F, Gregori G, et al. Quantifying choriocapillaris flow deficits using global and localized thresholding methods: a correlation study. Quant Imaging Med Surg. 2018 Dec;8(11):1102-12.

40 Chu Z, Cheng Y, Zhang Q, Zhou H, Dai Y, Shi Y, et al. Quantification of choriocapillaris with Phansalkar's local thresholding: pitfalls to avoid. Am J Ophthalmol. 2020;213:16176. 